This is the peer reviewed version of the following article: "Tutiv, C., Vidal, Y., Ancho, L., Rodellar, J. (2016). Hysteresis-based design of dynamic reference trajectories to avoid saturation in controlled wind turbines. Asian journal of control, (2), 19: 1-12." which has been published in final form at [doi: 10.1002/asjc.1383].. 


\title{
HYSTERESIS-BASED DESIGN OF DYNAMIC REFERENCE TRAJECTORIES TO AVOID SATURATION IN CONTROLLED WIND TURBINES †
}

\author{
Christian Tutivén, Yolanda Vidal, Leonardo Acho and José Rodellar
}

\begin{abstract}
The main objective of this paper is to design a dynamic reference trajectory based on hysteresis to avoid saturation in controlled wind turbines. Basically, the torque controller and pitch controller set-points are hysteretically manipulated to avoid saturation and drive the system with smooth dynamic changes. Simulation results obtained from a 5MW wind turbine benchmark model show that our proposed strategy has a clear added value with respect to the baseline controller (a well-known and accepted industrial wind turbine controller). Moreover, the proposed strategy has been tested in healthy condition but also in presence of a realistic fault where the baseline controller caused saturation to finally conduct to instability.
\end{abstract}

Key Words: Hysteresis, avoid saturation, wind turbine, super-twisting, fault tolerant control.

\section{INTRODUCTION}

In control systems, every actuator is prone to saturation due to its maximum and minimum physical limits. The analysis and design of a system that contains saturation nonlinearities, such as actuator saturation, is an important and challenging problem [1, 2, 3, 4, 5]. It is important that the control design takes into account the actuator limits to avoid undesirable effects such as transient response, degradation of the closed-loop performance, and even closed-loop instability [2]. In wind turbines (WTs) saturation can create limit cycles inducing dangerous vibrations on the WT structure.

In the literature, there have been efforts in addressing actuator saturation. For example, reference

\footnotetext{
Manuscript received 2016.

Christian Tutivén, Yolanda Vidal (corresponding author), Leonardo Acho, and José Rodellar are with the Mathematics Dept., Universitat Politècnica de Catalunya, \{christian.tutiven, yolanda.vidal, leonardo.acho, jose.rodellar $\} @$ upc.edu.

This work has been partially funded by the Spanish Ministry of Economy and Competitiveness through the projects DPI2014-58427-C2-1-R, DPI2015-64170-R (MINECO/FEDER), and by the Catalonia Government through the project 2014 SGR 859 .
}

[6] presents a synthesis of modern anti-windup techniques. A direct approach to deal with actuator saturation in wind turbines is used in [7] where an anti-windup controller to minimize the $H_{\infty}$ norm of the closed-loop system is designed. The design of a compensation method, which is based on variable structure systems to avoid both amplitude and rate input saturation by means of an auxiliary loop, is presented in [8]. In [9] the enlargement of the domain of stability of an actuator constrained state time-delay system with a novel dynamic two controller anti-windup design is proposed. It is also relevant the recent research on model predictive control in which constraint handling is given to cope with the actuator physical limitations, see $[10,11]$. Note that, in WT, some kind of faults can saturate the control action [12], and these saturation nonlinearities might lead to instability. Therefore, a robust system against saturation can better deal with faults.

Research into fault tolerant control (FTC) methods that allow WTs to continue operating in the presence of faults (at least during a reasonable time to take a corrective action [13]) are the crucial part to extend operating periods and thus minimize downtime and 
maximize productivity of WTs. FTC methods can be classified into two categories: passive and active methods [14]. The passive FTC takes into account some predefined fault modes in the design stage [15]. Hence, when the fault occurs, the controller is able to maintain the stability of the system with an acceptable degradation of its performance, and requires neither fault detection and diagnosis (FDD) systems nor a controller reconfiguration. In contrast, for active FTC it is indispensable to use a fault detection (FD) algorithm in order to react to the system failures actively by reconfiguring the controllers so that the stability and acceptable performance of the entire system can be maintained [16]. Recent years have seen a growing number of publications in the application of passive FTC to WTs. For example, a compensation technique for input constraints avoidance to the pitch control of a wind turbine is proposed in [8], and [17] proposes a new indirect adaptive fault-tolerant controller design method via state feedback for actuator fault compensation.

In this work, an avoid saturation control strategy is proposed based on reference adaptation ideas, using a hysteresis model in such a way that the actuator dynamics stay properly within the actuator physical limits. The motivation to use hysteresis to handle the controller set-point (to conduct the closed-loop system with a smooth behavior and to avoid saturation) comes from the fact that hysteresis incorporates smooth dynamics and history dependence. In particular, it incorporates the fact that the response for a decrease in the input variable is different from the response for an increase. The proposed strategy is tested in healthy condition but also under a fixed pitch angle measurement fault, thus showing the enhanced robustness of the closed loop system. Up to the authors knowledge, is the first time that hysteresis concept is used to derive dynamic reference trajectories in control loops.

The paper is divided into five sections. Section II presents general control concepts for WTs and introduces the selected 5MW benchmark WT used in the simulations. Also, the baseline control strategy for the Upwind 5MW wind turbine based on classical control methods and the controllers used in the proposed strategy (based on the super-twisting method [18]) are explained in this section. In section III the design of the proposed avoid saturation strategy is stated. In section IV the simulations results are presented and analyzed. Finally, section V provides the conclusions.
Table 1. WT Characteristics.

\begin{tabular}{lc}
\hline Rated power $\left(P_{n}\right)$ & $5 \mathrm{MW}$ \\
Number of blades & 3 \\
Height of tower & $87.6 \mathrm{~m}$ \\
Rotor diameter & $126 \mathrm{~m}$ \\
Cut in, Rated, Cut-Out Wind Speed & $3 \mathrm{~m} / \mathrm{s}, 11.4 \mathrm{~m} / \mathrm{s}, 25 \mathrm{~m} / \mathrm{s}$ \\
Gearbox ratio & 98 \\
Nominal generator speed $\left(\omega_{g, n}\right)$ & $1173.7 \mathrm{rpm}$ \\
\hline
\end{tabular}

\section{GENERAL CONCEPTS}

\subsection{Reference $5 \mathrm{MW}$ wind turbine}

In this work, the wind turbine given by the benchmark model in [19] is used. This benchmark utilizes FAST and thus proposes a higher-fidelity model of the WT, see [20]. The FAST code is an aerolastic simulator capable of predicting the extreme and fatigue loads of two and three bladed horizontal axis wind turbines. This simulator was developed by the National Renewable Energy Laboratory (NREL) and has been accepted by the scientific community and is used by many researchers in the development of new control systems for wind turbines, e.g. [21, 22, 23]. Numerical validation with FAST is performed with the NREL 5MW on-shore wind turbine. The wind turbine characteristics are summarized in Table 1, see [24].

In this work, the generator-converter and the pitch actuators are modeled and implemented externally; i.e. apart from the embedded FAST code. Thus, the next subsections will introduce these models as well as the generated wind data sets.

\subsection{Wind modeling}

In fluid dynamics, turbulence is a flow regime characterized by chaotic property changes [25]. This includes low momentum diffusion, high momentum convection, and rapid variation of pressure and velocity in space and time [26]. In the simulations, new wind data sets are generated in order to capture a more realistic turbulent wind simulation and, thus, to test the turbine controllers in a more realistic scenario. The turbulent-wind simulator TurbSim [27] developed by NREL is used and a full TurbSim wind field is employed in the simulations (Section IV). Fig. 1 shows the nominal downwind component of the hub-height wind velocity.

It can be seen from Fig. 1 that the wind speed covers the full load region (also called region 3 ) as its values range from $12.91 \mathrm{~m} / \mathrm{s}$ up to the maximum of $22.57 \mathrm{~m} / \mathrm{s}$. 


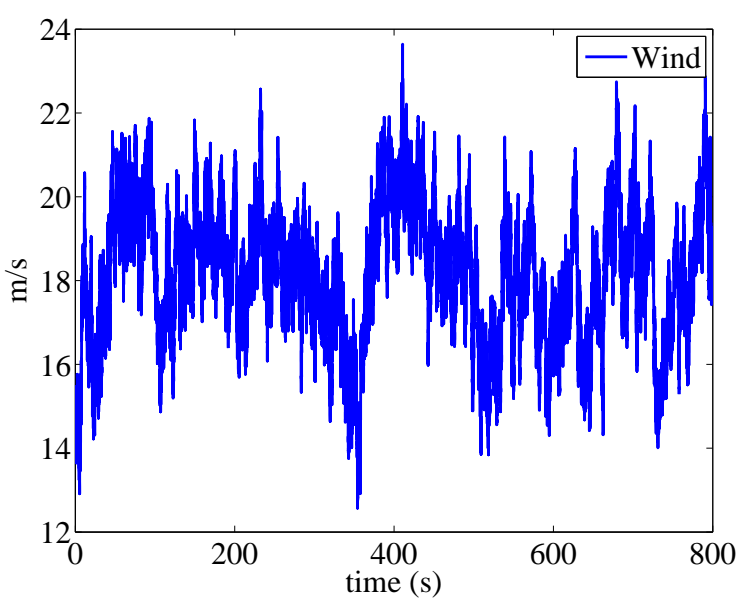

Fig. 1. Nominal downwind component of the hub-height wind velocity.

\subsection{Generator-converter actuator model}

The generator and converter dynamics can be modeled by a first-order differential system [28]

$$
\dot{\tau_{g}}(t)+\alpha_{g c} \tau_{g}(t)=\alpha_{g c} \hat{\tau}_{g, r}(t),
$$

where $\tau_{g}$ is the generator torque, $\hat{\tau}_{g, r}$ is the torque reference to the generator and $\alpha_{g c}$ is the generator and converter model parameter (in the simulations $\alpha_{g c}=50$ [24]). The electrical power produced by the generator can be modeled by

$$
P_{e}(t)=\eta_{g} \omega_{g}(t) \tau_{g}(t)
$$

where $\eta_{g}$ is the generator efficiency (in the simulations $\eta=0.98)$ and $\omega_{g}$ is the generator speed measurement.

\subsection{Pitch actuator model}

The hydraulic pitch system can be modelled by a second order system, see [19], with reference angle $\hat{\beta}_{c}$ and actual pitch angle $\beta_{i}$ as

$$
\ddot{\beta}_{i}(t)+2 \zeta \omega_{n} \dot{\beta}_{i}(t)+\omega_{n}^{2} \beta_{i}(t)=\omega_{n}^{2} \hat{\beta}_{c}(t),
$$

where $\zeta$ is the damping factor and $\omega_{n}$ is the natural frequency. This transfer function is associated to the pitch control system of every blade $(i=1,2,3)$. For the healthy case, the parameters $\zeta=0.6$ and $\omega_{n}=$ $11.11 \mathrm{rad} / \mathrm{s}$ are used [19].

\subsection{Baseline controllers}

The 5MW reference wind turbine given by FAST contains torque and pitch controllers for the full load region, see [24]. In this section we recall these controllers and refer to them as the baseline torque and pitch controllers, as they are used in the literature for comparison purposes, e.g. [21, 22, 29, 30, 31].

The torque and pitch controllers use the generator speed measurement as input. To mitigate highfrequency excitation of the control system, we filtered the generator speed measurement, using a recursive, single-pole low-pass filter with exponential smoothing as proposed by [24].

In the full load region, the torque controller maintains regulated the generated power, thus the generator torque is proposed inversely proportional to the filtered generator speed [20], or,

$$
\tau_{g, r}(t)=\frac{P_{\operatorname{ref}(t)}}{\hat{\omega}_{g}(t)},
$$

where $P_{\text {ref }}$ is the reference power (normally the nominal value is used), and $\hat{\omega}_{g}$ is the filtered generator speed. This controller will be referred as the baseline torque controller. As the generator may not be able to supply the desired electromechanic torque depending on the operating conditions, and in the case of overshooting, the torque controller is saturated to a maximum of $47402.9 \mathrm{Nm}$, rate limited to a maximum of $15000 \mathrm{Nm} / \mathrm{s}$ [24], and is recalled $\hat{\tau}_{g, r}$. It is important that the control design takes into account these actuator limits. Otherwise, undesirable effects can appear such as transient response, degradation of the closed-loop performance, and even closed-loop instability [2]. Obviously, all these degradation performance effects arrive from the saturation core, among other harmful events. Furthermore, saturation can create limit cycles inducing dangerous vibrations on the wind turbine structure.

To assist the torque control with regulating the wind turbine electrical power output, while avoiding significant loads and maintaining the rotor speed within acceptable limits, a collective pitch controller is added to the generator speed tracking error. The collective blade pitch gain scheduling PI-controller (GSPI) is one of the first well-documented controllers and it is used in the literature as a baseline controller to compare the obtained results. This GSPI control is a collective pitch controller that compensates the nonlinearities in the turbine by changing the controller gains according to a scheduling parameter. This controller was originally developed by Jonkman for the standard land-based 5MW turbine, see [24]. The GSPI control has the generator speed as input and the pitch servo set-point, $\beta_{c}$, as output. That is, 


$$
\begin{gathered}
\beta_{c}(t)=K_{p}(\gamma)\left(\hat{\omega}_{g}(t)-\omega_{g, r}(t)\right)+K_{i}(\gamma) \int_{0}^{t}\left(\hat{\omega}_{g}(t)-\omega_{g, r}(t)\right) d \tau \\
i=1,2,3
\end{gathered}
$$

where $\omega_{g, r}$ is the reference generator speed (usually the nominal value is used) and the scheduling parameter $\gamma$ is obtained by averaging the measurements of all pitch angles as

$$
\gamma=\frac{\sum_{i=1}^{3} \beta_{i}(t)}{3} .
$$

The scheduled gains are calculated following [24]. Finally, to not exceed the mechanical limitations of the pitch actuator, the input signal $\beta_{c}$ is saturated to a maximum of $90^{\circ}$ and a rate limit of $8^{\circ} / \mathrm{s}$ [24], and finally called $\hat{\beta}_{c}$.

\subsection{Super-Twisting algorithm controllers}

The torque controller used by the proposed strategy is the scalar super-twisting algorithm (STA) based controller [18]:

$$
\begin{aligned}
& \dot{y}(t)=-\alpha_{2} \operatorname{sgn}\left(P_{e}(t)-P_{\text {ref }}(t)\right)+\alpha_{3} a_{s s}(t), \\
& \tau_{g, r}(t)=-\alpha_{1} \sqrt{\left|P_{e}(t)-P_{\text {ref }}(t)\right|} \operatorname{sgn}\left(P_{e}(t)-P_{\text {ref }}(t)\right) \\
& +y(t),
\end{aligned}
$$

where $\alpha_{1}, \alpha_{2}, \alpha_{3}>0$ and $a_{s s}(t)$ is the side-to-side acceleration measured at the tower top. The stability analysis for this controller is given in [18]. Note that $\tau_{g, r}(t)$ is the torque control signal before the saturation and rate limiter actions (see Fig. 7).

We use the STA based pitch controller [18]:

$$
\begin{aligned}
& \dot{z}(t)=\operatorname{sgn}\left(\hat{\omega}_{g}(t)-\omega_{g, r}(t)\right)+\alpha_{4} a_{f a}(t), \\
& \beta_{c}(t)=K_{p}(\gamma)\left(\hat{\omega}_{g}(t)-\omega_{g, r}(t)\right)+K_{i}(\gamma) z(t),
\end{aligned}
$$

where $\alpha_{4}>0, a_{f a}(t)$ is the fore-aft acceleration measured at the tower top, and $\beta_{c}(t)$ is the collective pitch control before the saturation and rate limiter actions (see Fig. 7).

The STA-based torque and pitch controllers [18], compared to the baseline controllers, improve the overall performance of the WT in healthy and faulty conditions, and reduce the fore-aft and side-to-side accelerations, which is relevant in terms of vibration reduction. Under actuator saturation, the baseline and the STA-based controllers, both, may induce limit cycles. In this case, it leads to instability of the overall closed-loop system (a phenomenon also observed in controlled mechanical systems under saturation (see, for instance [32]).

The proposed saturation strategy can be used with any controller. In this work, it is used in conjunction with the baseline controllers and also with the STAbased controllers. Their respective performances are compared to the baseline controllers without any saturation strategy.

\section{DESIGN OF THE HYSTERESIS-BASED AVOID SATURATION STRATEGY}

Recall that WTs are basically controlled by manipulating the generator load torque (with the socalled torque control) and the blade pitch angles (with the so-called pitch control). These two controllers unitedly work to achieve the control objectives. Note that the main torque control objective is to regulate the electrical power and the main pitch control objective is to regulate the generator speed. This section describes the design of a strategy for saturation avoidance (SSA) based on a hysteresis law. Its main objective is to dynamically adapt the reference power $\left(P_{\text {ref }}\right)$ and the reference generator speed $\left(\omega_{g, r}\right)$ to avoid saturation and the consequent undesired effects, guaranteeing the closed-loop operation of the control system.

The first step is to define a so-called safety band (narrower than the band given by the actuator saturation limits) such that when the torque control signal leaves this band the reference power and generator speed are readjusted to bring back the torque control signal into the safety band. The safety band is defined by an upper torque value $\left(\tau_{u}\right)$ and a lower torque value $\left(\tau_{l}\right)$ given as follows and illustrated in Fig. 2,

$$
\tau_{u}=\tau_{n}+h, \quad \tau_{l}=\tau_{n}-h,
$$

where $\tau_{n}$ is the nominal torque and $h$ is defined as

$$
h=\frac{\tau_{\max }-\tau_{n}}{p},
$$

where $p>1$ is a parameter to be selected (the larger the value of $p$, the narrower will be the safety band) and $\tau_{\max }$ is the maximum allowable torque (saturation limit). Table 2 gives values of the above parameters, which will be used later on in the simulation results.

The second step is to introduce hysteretic loops relating the torque control signal, $\hat{\tau}_{g, r}$, with the desired reference power, $P_{\text {ref }}$, and the desired reference generator speed, $\omega_{g, r}$, respectively. With the purpose of 
Table 2. Values used by the SSA in the numerical simulations

\begin{tabular}{lc}
\hline$\tau_{\max }$ & $47.40 \mathrm{KNm}$ \\
$\tau_{n}$ & $40.68 \mathrm{KNm}$ \\
$\tau_{u}$ & $41.80 \mathrm{KNm}$ \\
$\tau_{l}$ & $39.55 \mathrm{KNm}$ \\
$\alpha$ & 0.5 \\
$p$ & 6 \\
$P_{n}$ & $5.00 \mathrm{MW}$ \\
$P_{u}$ & $5.97 \mathrm{MW}$ \\
$P_{l}$ & $4.07 \mathrm{MW}$ \\
$\omega_{g, n}$ & $122.91 \mathrm{rad} / \mathrm{s}$ \\
$\omega_{g, u}$ & $142.91 \mathrm{rad} / \mathrm{s}$ \\
$\omega_{g, l}$ & $102.91 \mathrm{rad} / \mathrm{s}$ \\
\hline
\end{tabular}

introducing the idea in a qualitative manner, Figures 2 and 3 are described to illustrate scenarios where the torque control leaves the safety band with either an increasing or decreasing tendency.

Point 1 in Fig. 2 indicates a time instant in which the torque control signal, $\hat{\tau}_{g, r}$, surpasses the upper torque value $\tau_{u}$. Then, the hysteretic loop, as indicated by point 1 in Fig. 3, readjusts the reference power, $P_{\text {ref }}$, to a slightly lower value (called $P_{l}$ ) with respect to the nominal one, $P_{n}$. A similar loop is considered for the reference generator speed, $\omega_{g, r}$, in Fig. 3 with the corresponding lower value $\omega_{g, l}$. These lower values for the reference signals drive the torque control signal back to the safety band region (see point 2 in Fig. 2 and Fig. 3).

On the other hand, when the torque control signal, $\hat{\tau}_{g, r}$, falls below the lower torque value, $\tau_{l}$, (see point 3 in Fig. 2) the reference power and the reference generator speed are adapted to slightly upper values with respect to the nominal ones, called $P_{u}$ and $\omega_{g, u}$ (see point 3 in Fig. 3). These increased values for the reference signals drive the torque control signal back to the safety band region (see point 4 in Fig. 2 and Fig. 3).

The lower values $\omega_{g, l}, P_{l}$ and the upper values $\omega_{g, u}, P_{u}$ are parameters of the SSA. In the simulations we use the values shown in Table 2.

One way to construct a hysteresis loop is through a special dynamic system described by a nonlinear differential equation. In this work, the following system is used

$$
\dot{z}(t)=\alpha[-z(t)+b \operatorname{sgn}(x(t)+a \operatorname{sgn}(z(t)))], \quad \alpha>0,
$$

where $a$ and $b$ are the hysteresis loop parameters shown in Fig. 4. This is a BIBO-stable system previously proposed in [33]. The transition speed between $b$ and $-b$ is governed by the positive parameter $\alpha$. With illustrative purposes, Fig. 5 shows the hysteretic loop

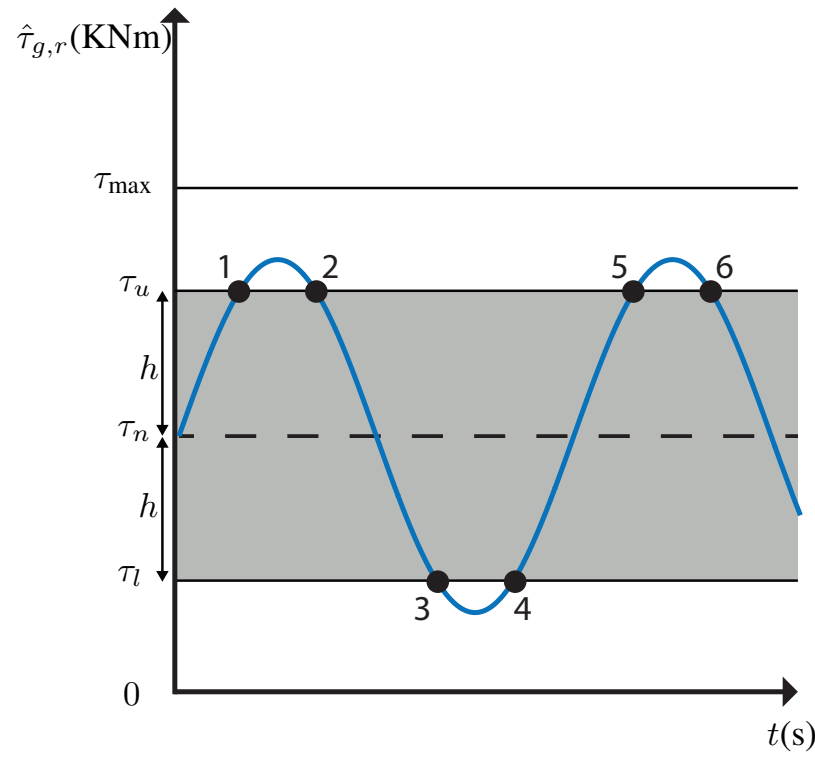

Fig. 2. Safety band defined by the upper torque value $\left(\tau_{u}\right)$ and the lower torque value $\left(\tau_{l}\right)$

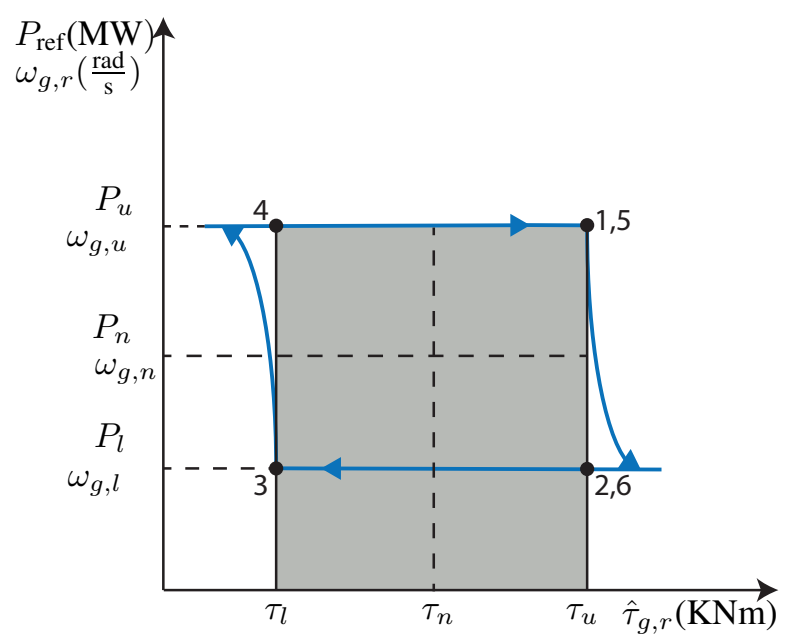

Fig. 3. Hysteresis-based reference signals readjustment.

obtained for the input $x=10 \sin (t)$ with parameters $a=b=1$, and $\alpha=10$. The vertical axis is $-z(t)$ instead of $z(t)$, in order to obtain an hysteresis loop with clock-wise orientation as proposed in Figure 3. Note that the hysteresis-loop generated by (10) is centered in the origin. However, torque values are centered around $\tau_{n}$, and set-point values, $P_{\text {ref }}$ and $\omega_{g, r}$, are centered around $P_{n}$ and $\omega_{g, n}$ respectively, see Fig. 3. Thus, in order to adapt this hysteretic system, a translation is needed as shown in Fig. 6. This translation is done by substracting the nominal torque, $\tau_{n}$, before the hysteresis block and adding the corresponding nominal 


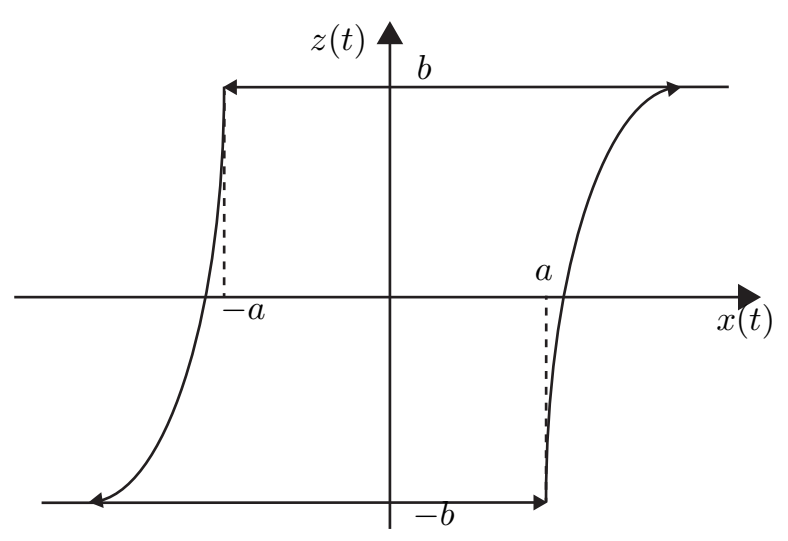

Fig. 4. Hysteresis behavior of system 10 .

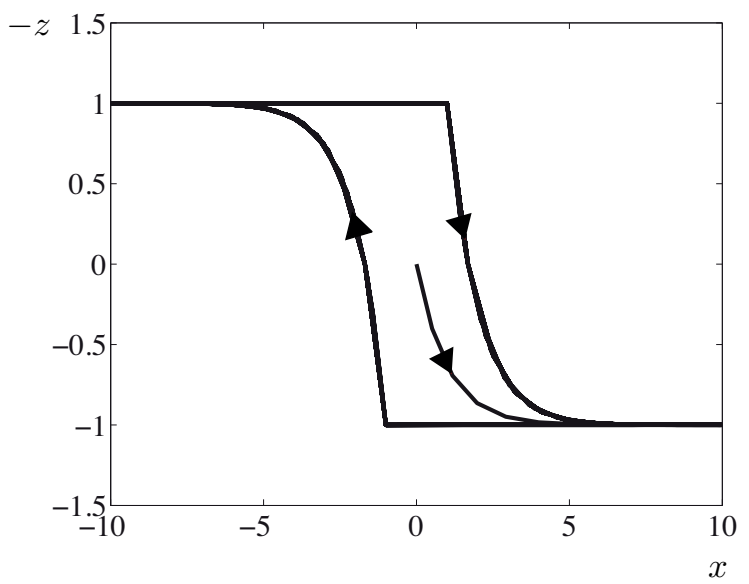

Fig. 5. Simulation results: $-z$ versus $x$.

reference values (power, $P_{n}$, and generator speed, $\omega_{g, n}$, respectively) after this block. Figure 6 depicts the final SSA block, where the input is the torque control signal and the outputs are the reference generator speed and the reference power.

Finally, Fig. 7 shows the closed-loop system for the $5 \mathrm{MW}$ benchmark wind turbine, including the generator, pitch actuator, torque and pitch STA controllers and integrating the newly proposed SSA block.

\section{Results}

The study compares the performance of the contributed SSA added to the baseline and also to the STA controllers, with respect to the standard baseline controller under two scenarios: fixed pitch measurement fault and healthy case.

The response of the electrical power, generator speed and accelerations at the tower top are analyzed

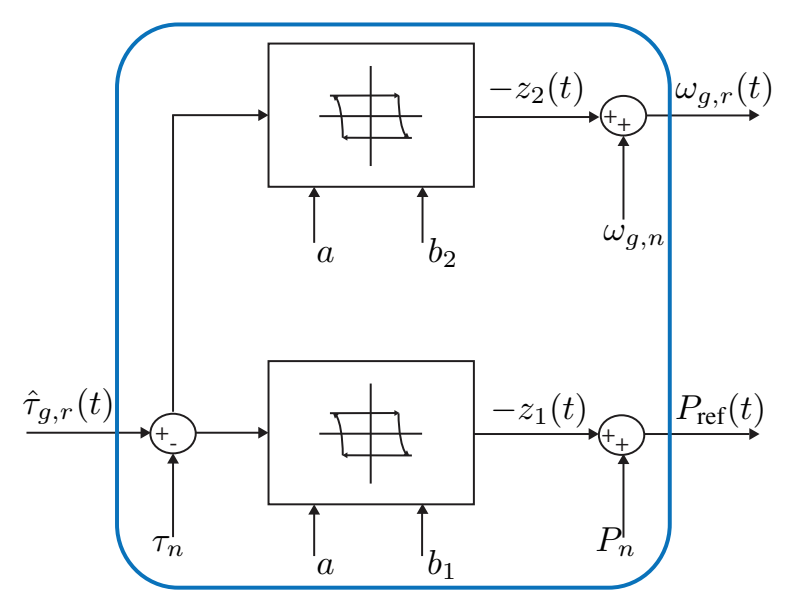

Fig. 6. Avoid saturation strategy (SSA) block $\left(a=1.12 \mathrm{KNm}, b_{1}=\right.$ $0.97 \mathrm{MW}, b_{2}=20.00 \frac{\mathrm{rad}}{\mathrm{s}}$ and $\left.\alpha=0.5\right)$.

in terms of the integral absolute error through the following performance indices:

$$
\begin{gathered}
J_{P}(t)=\int_{0}^{t}\left|P_{e}(\tau)-P_{n}\right| d \tau, \\
J_{\omega}(t)=\int_{0}^{t}\left|\omega_{g}(\tau)-\omega_{g, n}\right| d \tau, \\
J_{1}(t)=\int_{0}^{t}\left|a_{f a}(\tau)\right| d \tau, \\
J_{2}(t)=\int_{0}^{t}\left|a_{s s}(\tau)\right| d \tau,
\end{gathered}
$$

where $P_{n}$ is the rated power, $\omega_{g, n}$ is the nominal generator speed (see Table 2); $a_{f a}(t)$ is the fore-aft acceleration and $a_{s s}(t)$ is the side-to-side acceleration, both measured at the tower top.

\subsection{Fixed pitch angle measurement fault}

The origin of a possible fixed pitch angle measurement fault can be either electrical or mechanical [28]. Moreover, noise can be present in measurements. Thus, in the numerical simulations, random noise is added to the pitch measurements as proposed in [19]. This noise represents measurement noise either due to the measuring process or due to electrical noise in the system. In the simulation, the fault is introduced only to the third pitch angle measurement, $\beta_{3}$, which holds a constant fault value of $1 \mathrm{deg}$. (see [19]).

From Figures 8 (top) and 9 (top) we can observe that, with this fault, the standard baseline controllers (blue line) saturate and give rise to instability of the closed-loop system. On the other hand, the proposed SSA design, when used with the STA (green line) or the baseline controllers (red line), avoids saturation of 


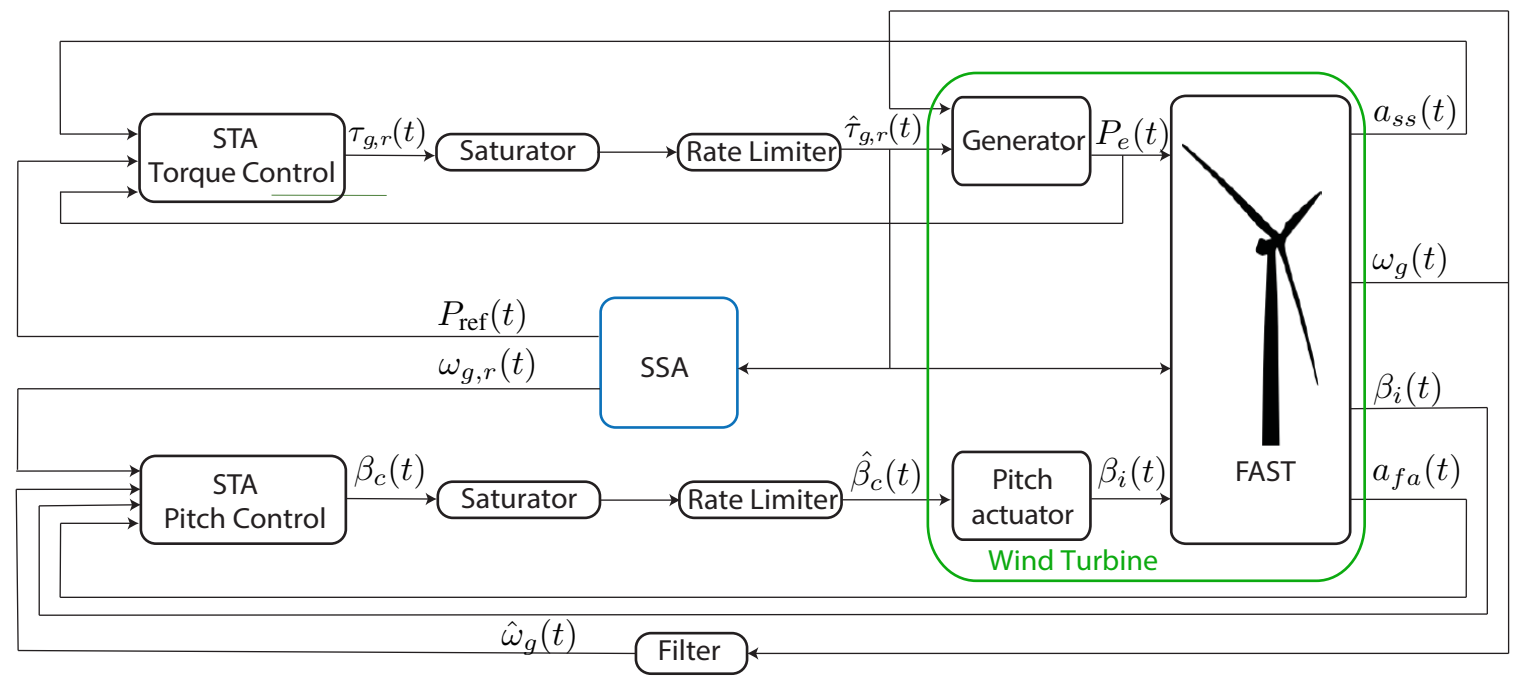

Fig. 7. Block diagram of the STA controllers with the added avoid saturation strategy (STA+SSA).
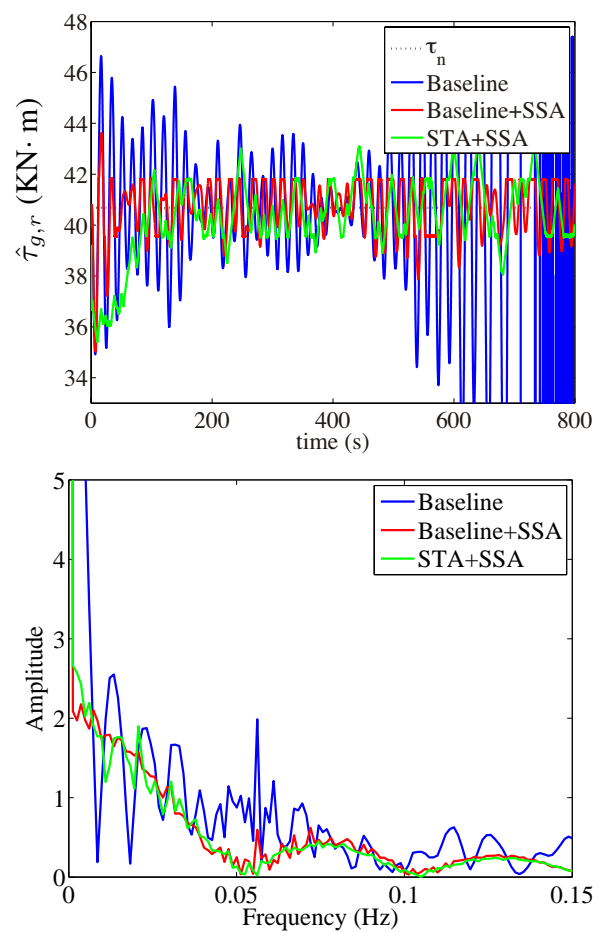

Fig. 8. Torque control (top), and its Fourier transform (bottom) with fixed pitch angle measurement.

$\tau_{g, r}(t)$, maintaining its values within the configured limits $\left(\tau_{u}\right.$ and $\left.\tau_{l}\right)$. Note that both torque and pitch control signals are smooth with a low dynamic activity (with respect to the standard baseline controllers) thanks to the used hysteresis system. This decreased actuators usage is relevant since it helps to increase the lifetime of these mechanical components. Moreover, according to Fig. 8 (bottom), the frequency harmonic content of the torque signal is also reduced, leading to a vibration attenuation in the WT structure. And according to Fig. 9, a similar performance is appreciated for the pitch angle control signal. On the other hand,
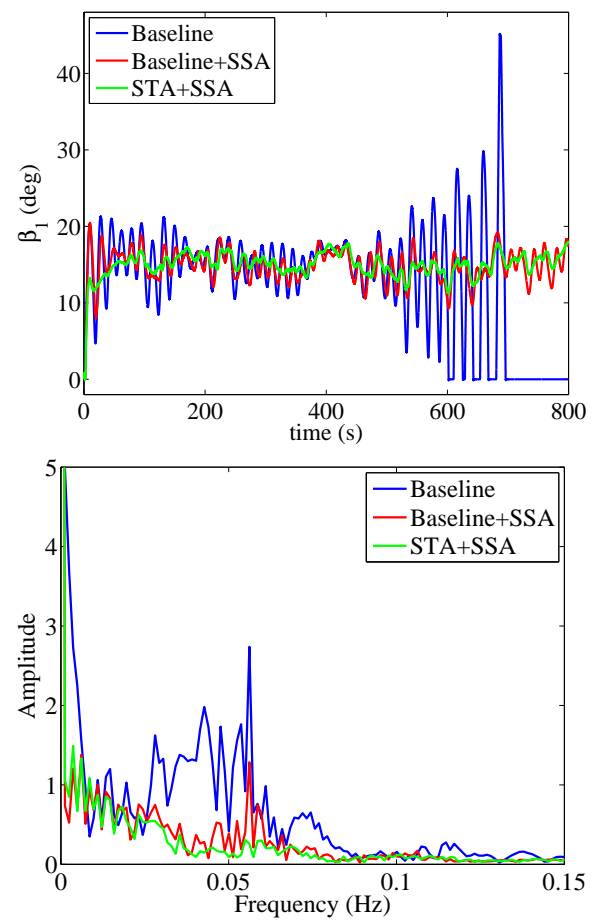

Fig. 9. Pitch angle $\beta_{1}$ (deg) (top), and its Fourier transform (bottom) with fixed pitch angle measurement fault. 
Figures 10 and 11 show that, with the proposed SSA strategy (when added to the baseline or STA controllers), the electrical power and generator speed are controlled close to the nominal value, even when the fault is present. Clearly, the standard baseline controller becomes unstable (blue line) for this type of fault.
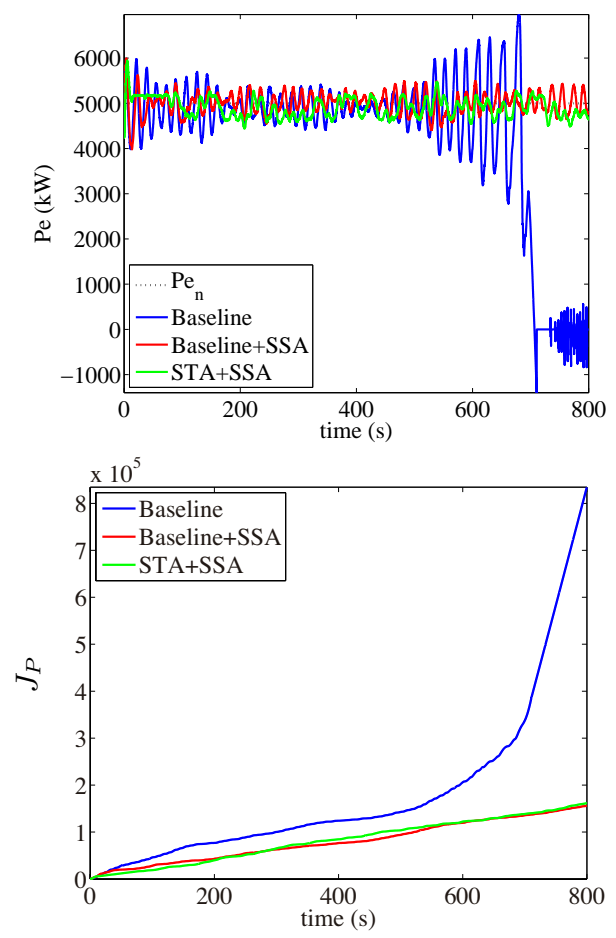

Fig. 10. Electrical power with fixed pitch angle measurement fault.

\subsection{Healthy Case}

The simulation results, previously discussed, have been also reproduced in a healthy scenario. From Figures 12 and 13, the electrical power and generator speed are correctly regulated with a similar performance for the three tested controllers: baseline, baseline plus SSA, and STA plus SSA.

Similarly to the faulty case, the proposed strategy has a smoother torque control performance, with respect to the baseline, maintaining its values within the configured limits ( $\tau_{u}$ and $\tau_{l}$ ), as can be observed in Fig. 14.

Recall that, when designing the pitch angle control loop, it is of great importance to avoid a high activity of the actuator, since it could not only lead to damage but also give rise to unstable modes of operation, see [34]. At this respect, the pitch control, shown in Fig. 15, is smoothed with the proposed strategy with respect to the baseline controllers.
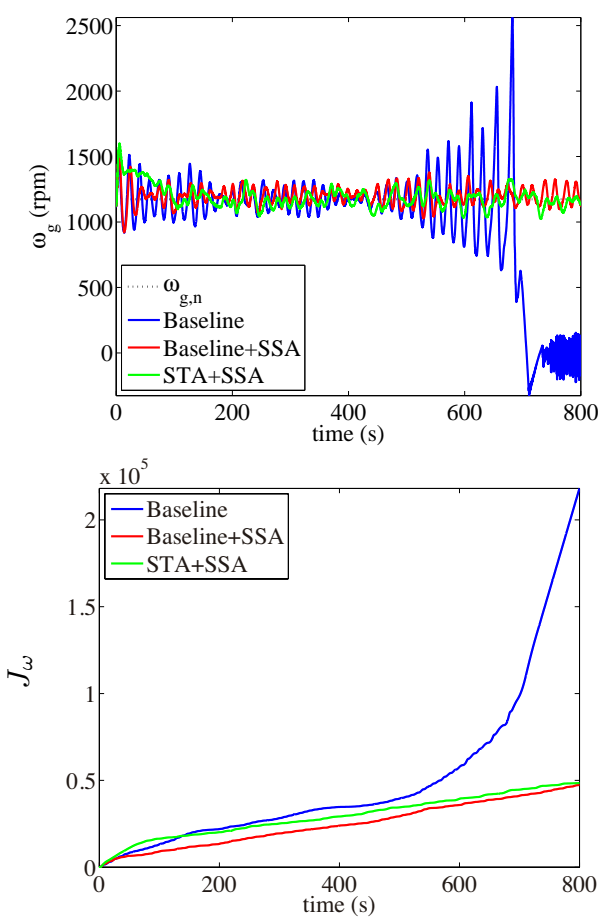

Fig. 11. Generator speed with fixed pitch angle measurement fault.
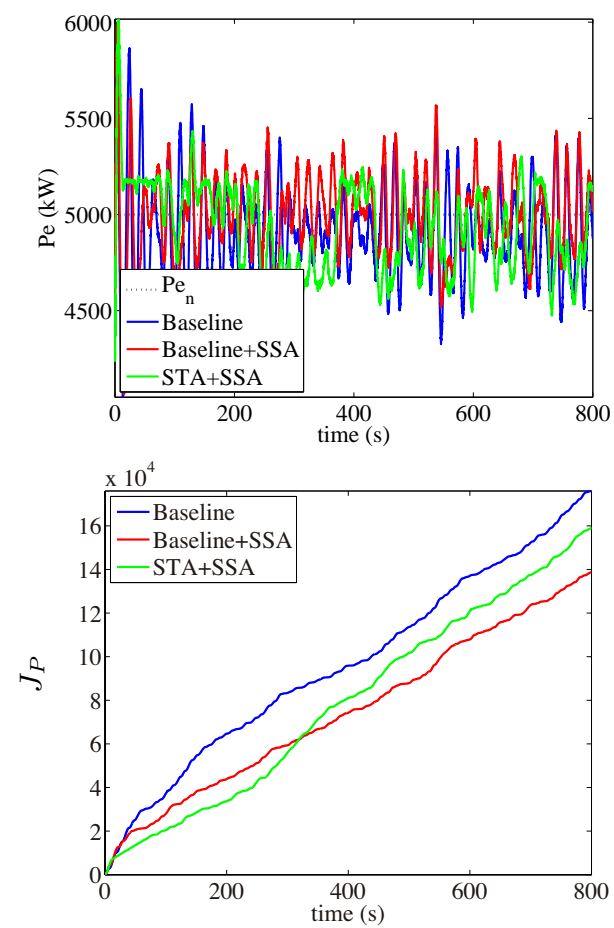

Fig. 12. Electrical Power (healthy case).

Lastly, due to the fact that the torque and pitch 
Table 3. Influence of narrowing the safety band on the operation and the performance of the WT: mean and standard deviation.

\begin{tabular}{lc|cccc|cccc} 
& & \multicolumn{5}{|c|}{ mean } & \multicolumn{4}{c}{ std. deviation } \\
\hline Controller & $p$ & $P e$ & $\omega_{g}$ & $\tau_{c}$ & $\beta$ & $P e$ & $\omega_{g}$ & $\tau_{c}$ & $\beta$ \\
\hline Baseline & - & 4900.7 & 1175.4 & 40.7 & 15.1 & 260.6 & 81.5 & 1.7 & 2.3 \\
Baseline+SSA & 6 & 5004.9 & 1199.8 & 40.7 & 14.8 & 223.4 & 68.5 & 1.1 & 2.0 \\
Baseline+SSA & 10 & 4903.7 & 1187.7 & 40.3 & 15.0 & 208.2 & 67.9 & 1.1 & 1.9 \\
\hline
\end{tabular}

Table 4. Influence of narrowing the safety band on the operation and the performance of the WT: performance indices.

\begin{tabular}{lccccc} 
Controller & $p$ & $J_{p}$ & $J_{w}$ & $J 1$ & $J 2$ \\
\hline Baseline & - & $1.7 \mathrm{e}+05$ & $4.9 \mathrm{e}+04$ & 74.1 & 49.2 \\
Baseline+SSA & 6 & $1.3 \mathrm{e}+05$ & $4.3 \mathrm{e}+04$ & 69.4 & 41.6 \\
Baseline+SSA & 10 & $1.4 \mathrm{e}+05$ & $4.2 \mathrm{e}+04$ & 70.8 & 40.1 \\
\hline
\end{tabular}
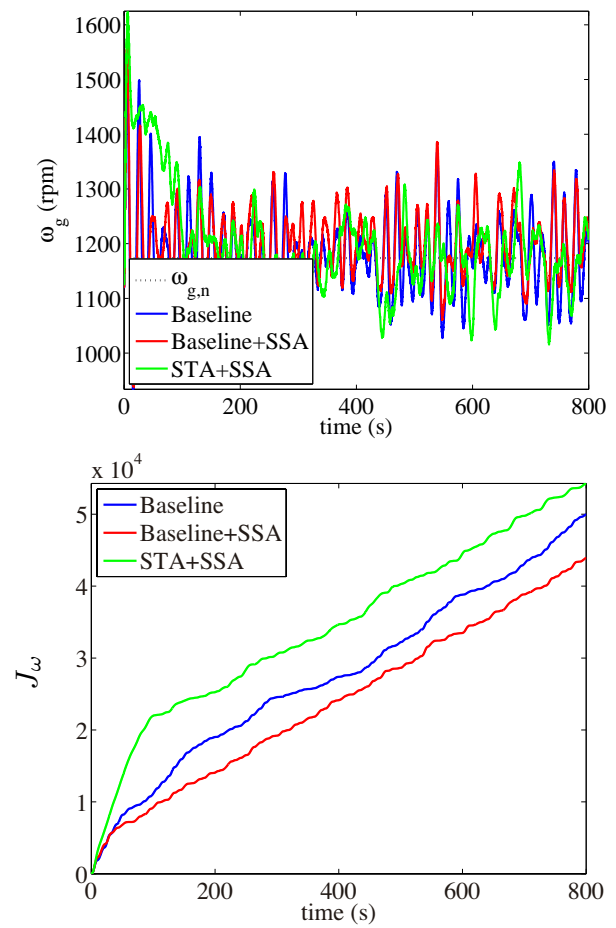

Fig. 13. Generator speed (healthy case).

actions are softer, this strategy reduces the accelerations on the tower (see Figures 16 and 17). In particular $J_{1}(t)$ (related to fore-aft accelerations) and $J_{2}(t)$ (related to side-to-side accelerations) indices have been reduced with respect to the standard baseline controllers.

Tables 3 and 4 show the influence of narrowing the safety band on the operation and the performance of the WT. Comparison between the baseline controller and different values of $p$ used in the baseline plus SSA show that the standard deviation of electrical power, generator speed, torque control and pitch angle are dramatically
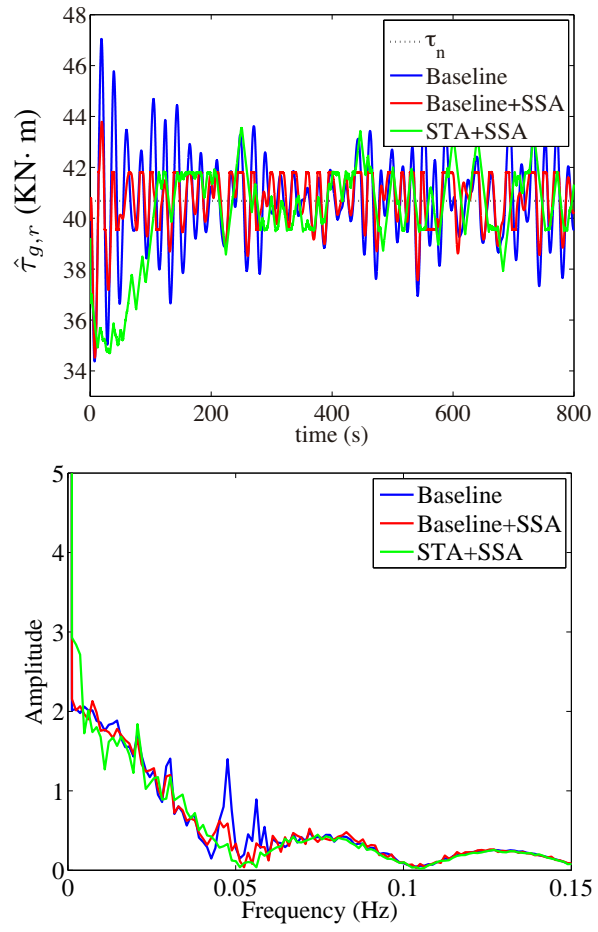

Fig. 14. Torque control (top), and its Fourier transform (bottom) in healthy case.

reduced with the proposed strategy, while mean values remain similar (see table 3). Table 4 shows that the proposed strategy improves all the performance indices, however increasing $p$ (narrowing the safety band) does not lead always to a reduction in all the performance indices. Thus, to determine the optimal $p$ value an optimization problem should be solved predefining an objective functional to be minimized. This is beyond the scope of this paper.

\section{Conclusions}

The main contribution of this work is a novel avoid saturation strategy (up to the authors knowledge, hysteresis loops have not been previously considered in this context), which also reduces significantly the torque and pitch actuators activity as well as the fore-aft and 

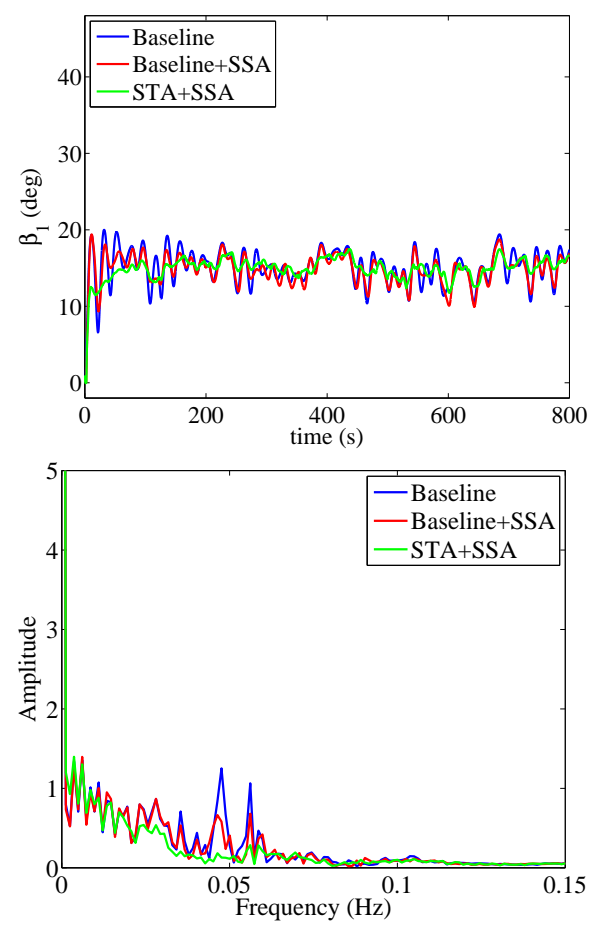

Fig. 15. Pitch angle $\beta_{1}(\mathrm{deg})$, and its Fourier transform (bottom) in healthy case.
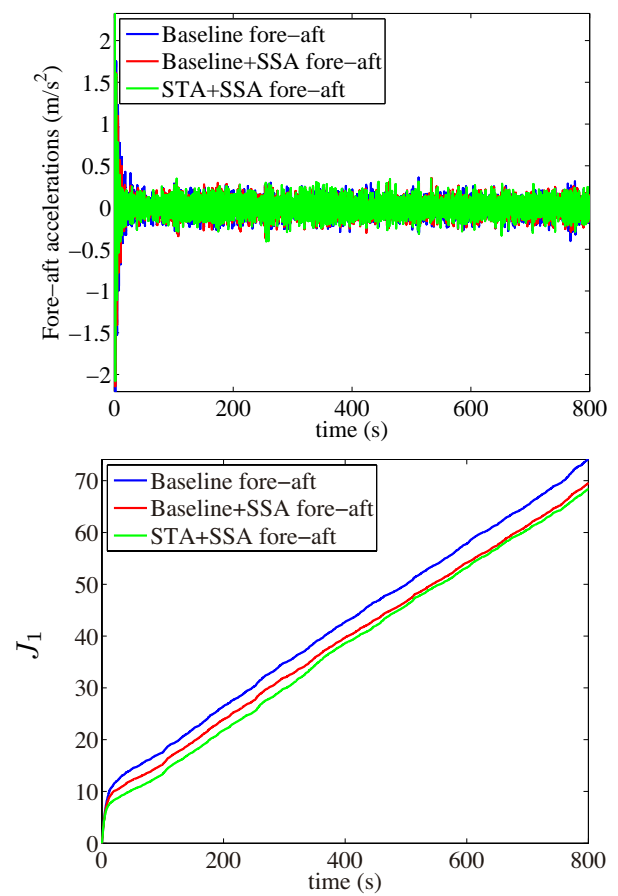

Fig. 16. Fore-aft accelerations at top tower (healthy case).

side-to-side accelerations at the tower top (with respect
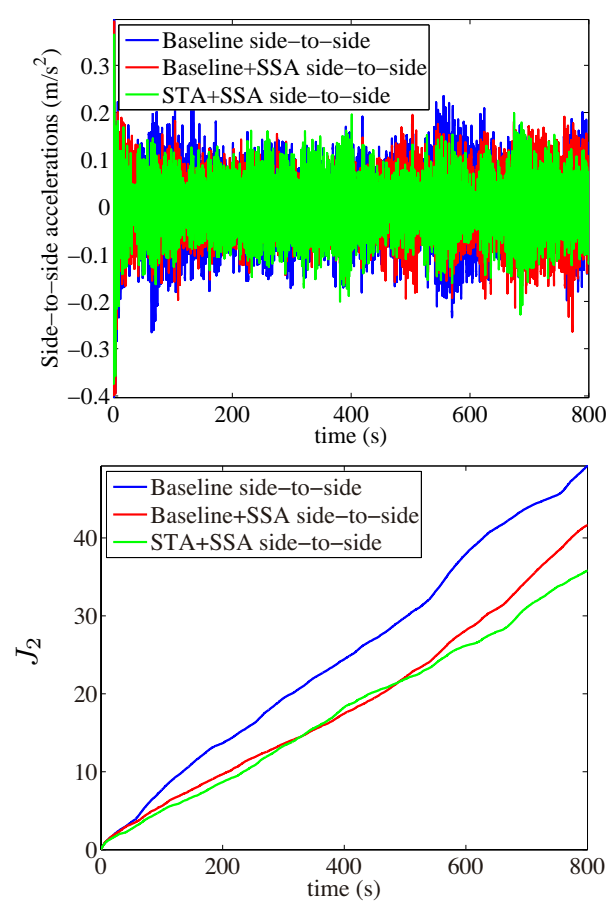

Fig. 17. Side-to-side accelerations at top tower (healthy case).

to the baseline controllers). That is, lower activity of the torque and pitch actuators is obtained, thus bringing on an extended lifetime of the actuators. Moreover, according to the numerical results, the overall closedloop system is robust against the studied fault.

Although the avoid saturation strategy has been proposed within a control scheme and a control methodology for wind turbines, the concept can also be applied in other control problems and along with a variety of control methodologies where the design of dynamic reference trajectories with memory capabilities can be advisable.

\section{REFERENCES}

1. T. Hu, Z. Lin, Control systems with actuator saturation: analysis and design, Springer Science \& Business Media, 2001.

2. V. Kapila, K. Grigoriadis, Actuator saturation control, CRC Press, 2002.

3. Y.-Y. Cao, Z. Lin, Robust stability analysis and fuzzy-scheduling control for nonlinear systems subject to actuator saturation, Fuzzy Systems, IEEE Transactions on 11 (1) (2003) 57-67.

4. Y.-F. Li, Design of $\mathrm{H}_{\infty}$ static output feedback control for discrete-time systems with limited actuator, Asian Journal of Control 17 (1) (2015) 284-296. 
5. J. Luo, J. Zhao, Robust control for a class of uncertain switched fuzzy systems with saturating actuators, Asian Journal of Control 17 (4) (2015) 1462-1469.

6. L. Zaccarian, A. R. Teel, Modern anti-windup synthesis: control augmentation for actuator saturation, Princeton University Press, 2011.

7. M. Meisami-Azad, K. M. Grigoriadis, Antiwindup linear parameter-varying control of pitch actuators in wind turbines, Wind Energy 18 (2) (2015) 187-200.

8. F. Garelli, P. Camocardi, R. J. Mantz, Variable structure strategy to avoid amplitude and rate saturation in pitch control of a wind turbine, International Journal of Hydrogen Energy 35 (11) (2010) 5869-5875.

9. A. Ahmed, M. U. Khan, M. Rehan, N. Iqbal, Two-controller anti-windup design for enlarging domain of stability of actuator constrained statedelay systems, Asian Journal of Control 15 (6) (2013) 1821-1832.

10. L. C. Henriksen, M. H. Hansen, N. K. Poulsen, Wind turbine control with constraint handling: a model predictive control approach, Control Theory \& Applications, IET 6 (11) (2012) 17221734.

11. D. Q. Mayne, Model predictive control: Recent developments and future promise, Automatica 50 (12) (2014) 2967-2986.

12. C. Edwards, T. Lombaerts, H. Smaili, Fault tolerant flight control, Lecture Notes in Control and Information Sciences 399, 2010.

13. V. Puig, J. Quevedo, T. Escobet, B. Morcebo, C. Ocampo, Control tolerante a fallos: Fundamentos y diagnóstico de fallos, Revista Iberoamericana de Automática e Informática Industrial 1 (1) (2004) 15-31.

14. J. Jiang, Fault-tolerant control systems: An introductory overview, Acta Automatica Sinica 31 (1) (2005) 161-174.

15. F. Guenab, P. Weber, D. Theilliol, Y. Zhang, Design of a fault tolerant control system incorporating reliability analysis and dynamic behaviour constraints, International Journal of Systems Science 42 (1) (2011) 219-233.

16. J. Jiang, X. Yu, Fault-tolerant control systems: A comparative study between active and passive approaches, Annual Reviews in control 36 (1) (2012) 60-72.

17. G.-H. Yang, D. Ye, Adaptive fault-tolerant $\mathrm{H}_{\infty}$, control via state feedback for linear systems against actuator faults, in: Decision and Control,
2006 45th IEEE Conference on, IEEE, 2006, pp. 3530-3535.

18. C. Tutivén, Y. Vidal, J. Rodellar, L. Acho, Acceleration-based fault tolerant control design of offshore fixed wind turbines, Structural Control and Health Monitoring (Submitted).

19. P. F. Odgaard, K. E. Johnson, Wind turbine fault detection and fault tolerant control-an enhanced benchmark challenge, in: Proceedings of American Control Conference, IEEE, 2013, pp. 4447-4452.

20. J. M. Jonkman, M. L. Buhl Jr, Fast users guide, National Renewable Energy Laboratory, Golden, CO, Technical Report No. NREL/EL-500-38230.

21. D. S. Ochs, R. D. Miller, W. N. White, Simulation of electromechanical interactions of permanentmagnet direct-drive wind turbines using the fast aeroelastic simulator, Sustainable Energy, IEEE Transactions on 5 (1) (2014) 2-9.

22. Y. Vidal, L. Acho, N. Luo, M. Zapateiro, F. Pozo, Power control design for variable-speed wind turbines, Energies 5 (8) (2012) 3033-3050.

23. B. Beltran, M. El Hachemi Benbouzid, T. AhmedAli, Second-order sliding mode control of a doubly fed induction generator driven wind turbine, Energy Conversion, IEEE Transactions on 27 (2) (2012) 261-269.

24. J. Jonkman, S. Butterfield, W. Musial, G. Scott, Definition of a $5 \mathrm{MW}$ reference wind turbine for offshore system development, Tech. rep., National Renewable Laboratory (NREL), Golden, Colorado (2009).

25. P. Davidson, et al., Turbulence: an introduction for scientists and engineers, Oxford University Press, USA, 2015.

26. H. K. Versteeg, W. Malalasekera, An introduction to computational fluid dynamics: the finite volume method, Pearson Education, 2007.

27. N. Kelley, B. Jonkman, NWTC computer-aided engineering tools (Turbsim), URL http://wind. nrel. gov/designcodes/preprocessors/turbsim.

28. P. F. Odgaard, J. Stoustrup, M. Kinnaert, Faulttolerant control of wind turbines: A benchmark model, IEEE Transactions on Control Systems Technology 21 (4) (2013) 1168-1182.

29. B. Beltran, T. Ahmed-Ali, M. Benbouzid, Highorder sliding-mode control of variable-speed wind turbines, Industrial Electronics, IEEE Transactions on 56 (9) (2009) 3314-3321. doi:10.1109/TIE.2008.2006949.

30. R. Chaaban, D. Ginsberg, C. Fritzen, Structural load analysis of floating wind turbines under 
blade pitch system faults, in: N. Luo, Y. Vidal, L. Acho (Eds.), Wind Turbine Control and Monitoring, Advances in Industrial Control, Springer International Publishing, 2014, pp. 301 -334 .

31. A. Diaz de Corcuera, A. Pujana-Arrese, J. Ezquerra, A. Milo, J. Landaluze, Design of robust controllers for load reduction in wind turbines, in: N. Luo, Y. Vidal, L. Acho (Eds.), Wind Turbine Control and Monitoring, Advances in Industrial Control, Springer International Publishing, 2014, pp. 97-133. doi:10.1007/978-3-319-08413-8_5.

32. T. L. Vincent, W. J. Grantham, [Page 102] Nonlinear and optimal control systems, John Wiley \& Sons, 1997.

33. L. Acho, Y. Vidal, Hysteresis modeling of a class of RC-OTA hysteretic-chaotic generators, 5th International Conference on Physics and Control, León, Spain, 2011.

34. F. D. Bianchi, H. De Battista, R. J. Mantz, Wind turbine control systems: principles, modelling and gain scheduling design, Springer Science \& Business Media, 2006. 